\title{
Simulation Research on Airflow Field of Bulldozer Cab
}

\author{
Zhu Jian, Ren Hongjuan, Luo Yiping \\ College of Automobile Engineering, Shanghai University of Engineering Science, Shanghai, P. R. China \\ Email address: \\ 371754791@qq.com (Zhu Jian), 122587984@qq.com (Ren Hongjuan), Lyp777@sina.com (Luo Yiping)
}

To cite this article:

Zhu Jian, Ren Hongjuan, Luo Yiping. Simulation Research on Airflow Field of Bulldozer Cab. International Journal of Mechanical

Engineering and Applications. Vol. 4, No. 4, 2016, pp. 152-155. doi: 10.11648/j.ijmea.20160404.13

Received: June 20, 2016; Accepted: June 30, 2016; Published: July 23, 2016

\begin{abstract}
This paper used the airflow organizations of a bulldozer cab as the research object, and the physical model and mathematical model of the three-dimensional airflow is played, and computational fluid dynamic software FLUENT is used to simulate the airflow organizations. The governing equations were solved by using the $R N G k-\varepsilon$ two equation turbulence model and SIMPLE algorithm. We obtained the simulation results of temperature field and velocity field distribution of different air-supply modes in the driving room of the bulldozer in summer. The results show airflow distribution by different types of air-supply of the air conditioning of the bulldozer cab, which provides a theoretical basis to improve the thermal environment, and it has guiding function to the design of air duct of the engineering vehicle.
\end{abstract}

Keywords: Bulldozer, Air Distribution, Velocity Field, Temperature Field

\section{Introduction}

Vehicle engineering played an irreplaceable role in the country's infrastructure. With the development of air conditioning of automotive, engineering vehicles with air conditioning could improve working conditions. The study on the flow and heat transfer of the air in the vehicle was the basis of the air flow organization design and the evaluation of the comfortable environment [1]. Scholars at home and abroad actively carried out research in this area, and some achievements have been obtained. CURRLE $\mathrm{J}$ et al. studied the influence of airflow, velocity and temperature of the flow parameters and outlet area on the local and overall thermal comfort of the occupants by the method of numerical simulation [2]. Aronson Dag et al. simulated the distribution of velocity field of the cab, and compared with the PIV test results, the comparison was consistent [3]. Chen Jiangping et al. made ventilation duct system of the air conditioning into the calculation area of the car room, and simulated the distribution of the flow field and temperature field in the vehicle [4]. Ye Xiaojiang et al. simulated the three-dimensional airflow field of air conditioned train, and provided the basis for the optimization of the comfortable environment of air conditioned cars [5]. Lu Kelong et al. made air conditioning system and the passenger compartment as a whole according to the thermal comfort of occupants, which improved the air duct and the thermal comfort of the driver [6].

Although there were a lot of researches about the flow field analysis of air conditioning of automotive, but most of them focused on the speed field of the passenger compartment. The research on the flow field of the engineering vehicle was very few. In this paper, we used bulldozer as a research object. We also added human body model, and used the second boundary condition to simulate velocity field and temperature field of airflow of the bulldozer cab by ceiling air-supply and the side wall air-supply.

\section{Mathematical Model}

\subsection{Basic Control Equation}

In the car flow field, the air movement speed was relatively small, and the density of the air change was not big, so the air in the cab could be seen as a three-dimensional incompressible flow field. Reynolds number was relatively small, but it was still more than the critical Reynolds number, the flow field was treated as a steady-state turbulent model [7, 8]. At the same time without considered the impact of air leakage, we could consider the car have good air tightness. Due to the radiation of the solar radiation and the solid surface in the vehicle interior, the airflow in the vehicle was a 
radiation transparent medium.

The basic governing equations were expressed as follows:

1) Continuous equation

$$
d i \vec{v}=\mathbf{0}
$$

2) Momentum equation

$$
\operatorname{div}\left(p \vec{v} v_{i}-\mu_{e f f} g r a d v_{i}\right)=-\frac{\partial(\rho)}{\partial x_{i}}+\operatorname{div}\left(\mu_{e f f} \frac{\partial \vec{v}}{\partial x_{i}}\right)
$$

3) Energy equation

$$
\frac{\partial(\rho T)}{\partial T}+\operatorname{div}(\rho \vec{v} T)=\operatorname{div}\left(\frac{k}{c_{p}} \operatorname{grad} T\right)+S_{T}
$$

4) Turbulent kinetic energy equation

$$
\operatorname{div}\left(\rho \vec{v} \kappa-\Gamma_{\kappa e f f} \operatorname{grad} \kappa\right)=G-\rho \varepsilon
$$

5) Turbulent kinetic energy dissipation rate equation

$$
\begin{gathered}
\operatorname{div}\left(\rho \vec{v} \varepsilon-\Gamma_{\varepsilon e f f} g r a d \varepsilon\right)=\frac{\varepsilon}{\kappa}\left(C_{1} G-C_{2} \rho \varepsilon\right) \\
G=\mu_{e f f}\left[2 \sum_{i=1}^{3}\left(\frac{\partial v_{i}}{\partial x_{i}}\right)^{2}+\left(\frac{\partial \mu}{\partial y}+\frac{\partial v}{\partial x}\right)^{2}+\left(\frac{\partial v}{\partial z}+\frac{\partial w}{\partial y}\right)^{2}+\left(\frac{\partial w}{\partial x}+\frac{\partial \mu}{\partial z}\right)^{2}\right]
\end{gathered}
$$

$\vec{v}$ stands for average velocity; $v_{i}$ stands for velocity component; $x_{i}$ stands for Coordinate component; $\mathrm{T}$ stands for temperature; $k$ stands for Heat transfer coefficient of fluid; $C_{p}$ stands for Specific heat; $S_{T}$ stands for Internal heat source of fluid; $\kappa$ stands for Turbulent kinetic energy; $\varepsilon$ stands for Turbulent kinetic energy dissipation rate; $\mu$ stands for Effective viscosity coefficient of turbulent flow; $\rho$ stands for Air density, it is $1.16 \mathrm{~kg} / \mathrm{m}^{3}$ in the room temperature; $\Gamma_{\kappa e f f}$ stands for Effective diffusion coefficient of turbulent kinetic energy; $\Gamma_{\varepsilon e f f}$ stands for effective diffusion coefficient of Turbulent kinetic energy viscous dissipation rate.

\subsection{Selection of RNGk-E Turbulence Model}

Although there were many kinds of turbulence models, it is widely used in the field of indoor flow field were Turbulence model and Zero equation turbulent model. The Zero equation turbulence model was the most simple turbulence model. It could be used for solving problems in geometry and flow characteristics. Although the model calculation was relatively fast, high efficiency, at the same time, the computer resource requirements was not high, but if the flow was obvious separation and recirculation, the turbulence model was no longer obtained accurate results [9].

Because the turbulence model could better deal with the high strain rate and large flow, so the turbulence model has a high accuracy in the indoor flow field simulation and the airflow organization. So in this paper, we used the $R N G k-\varepsilon$ turbulence model, and the wall function method was adopted in the cohesive layer in the vicinity of a wall.

Turbulence model was based on statistical principle derived the transient Navier Stokes equations by Renormalization Group of mathematical methods, through the large scale motion and amendment of viscosity reflected the influence of small scale, and removed these small scale motion system from the control equation $[9,10]$.

The $R N G k-\varepsilon$ turbulent kinetic energy and dissipation rate equations were similar to the standard model, but the $R N G k-\varepsilon$ turbulence model was modified and accounted for the rotation of the flow.

The control equations of the $R N G k-\varepsilon$ turbulence model are as follows:

$$
\begin{gathered}
\rho \frac{D k}{D t}=\frac{\partial}{\partial x_{i}}\left[\left(\alpha_{k} \mu_{e f f}\right) \frac{\partial k}{\partial x_{i}}\right]+G_{k}+G_{b}-\rho \varepsilon-Y_{M} \\
\rho \frac{D \varepsilon}{D t}=\frac{\partial}{\partial x_{i}}\left[\left(\alpha_{\varepsilon} \mu_{e f f}\right) \frac{\partial \varepsilon}{\partial x_{i}}\right]+C_{1 \varepsilon} \frac{\varepsilon}{k}\left(G_{k}+C_{3 \varepsilon} G_{b}\right)-C_{2 \varepsilon} \rho \frac{\varepsilon^{2}}{k}-R
\end{gathered}
$$

Parameter $G_{k}$ is generated by turbulent kinetic energy which caused by the average velocity gradient; $Y_{M}$ stands for the influence of the pressure velocity turbulent pulsation expansion on the total dissipation rate. At this point, it is $0 ; \alpha_{k}$ and $\alpha_{\varepsilon}$ are reciprocal number of effective turbulent prandtl number of turbulent kinetic energy and dissipation rate.

\section{Research Model of Engineering Vehicle Cab}

\subsection{Cab Structure}

This paper used a large bulldozer cab for the research object, and established side wall air-supply and ceiling air-supply of construction vehicle cab model. Cold air through the front fixed inlet tuyere and side wall (roof) into the cab, and the airflow discharged form the car by the outlet on side wall. The concrete structure was shown in Fig. 1.

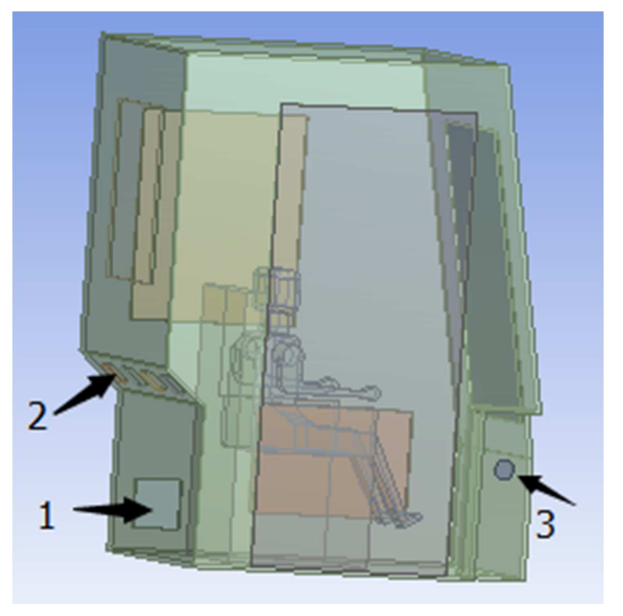




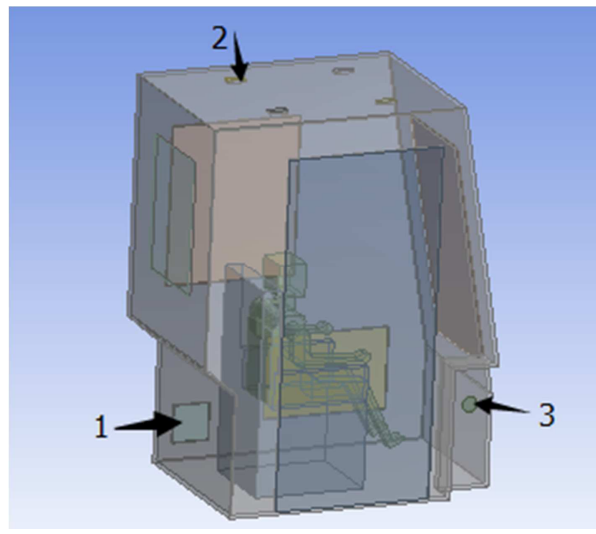

1-outlet, 2-side wall (roof) inlet, 3 -fixed inlet

Fig. 1. Cab structure of bulldozer.

\subsection{Calculation Model of Numerical Simulation}

The simulation of two equation turbulence model of cap airflow field was adopted in $R N G k-\varepsilon$ model, boundary conditions setting the velocity inlet, and wind speed was 4 $\mathrm{m} / \mathrm{s}$. The supply air temperature was $19^{\circ} \mathrm{C}$. Temperature of ambient and cab wall were $39^{\circ} \mathrm{C}$. Made the driver as heat source which valued $100 \mathrm{~W} / \mathrm{m}^{2}$ [11]. Considered the external glass radiation and wall heat transfer.

Grid generation technique was an essential part of computational fluid dynamics (CFD). The time which mesh generation required accounts for a $60 \%$ of all tasks of human time and CFD calculation precision in a large extent dependent on the generated grid quality. Thus, the grid generation technique was one of the key technologies in the computational fluid dynamics. In order to simulate the complex flow field, the gradient of the flow parameter which was changed larger need setting more grid point. That is to say the physical condition of the more complex areas, the grid should be properly encrypted. In this paper, the unstructured tetrahedral mesh of Fluent software was used to partition the cab, and the number was 336778 , as shown in Fig. 2. Used Fluent software to calculate the pressure velocity coupling using SIMPLE algorithm, and the discrete calculation of the region used the two order upwind scheme.

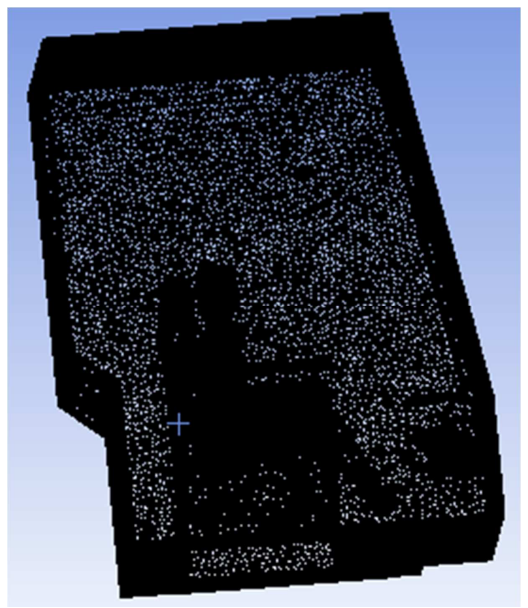

Fig. 2. The grid of Bulldozer cab.

\section{Simulation Result Analysis}

In this paper, we got air flow and heat transfer conditions of the bulldozer cab by Fluent finite simulation calculation. Fig. 3 was shown the cloud map of velocity field of two wind -supply forms of the side wall / top. Fig. 4 was shown the cloud map of temperature field of two wind -supply forms of the side wall / top.
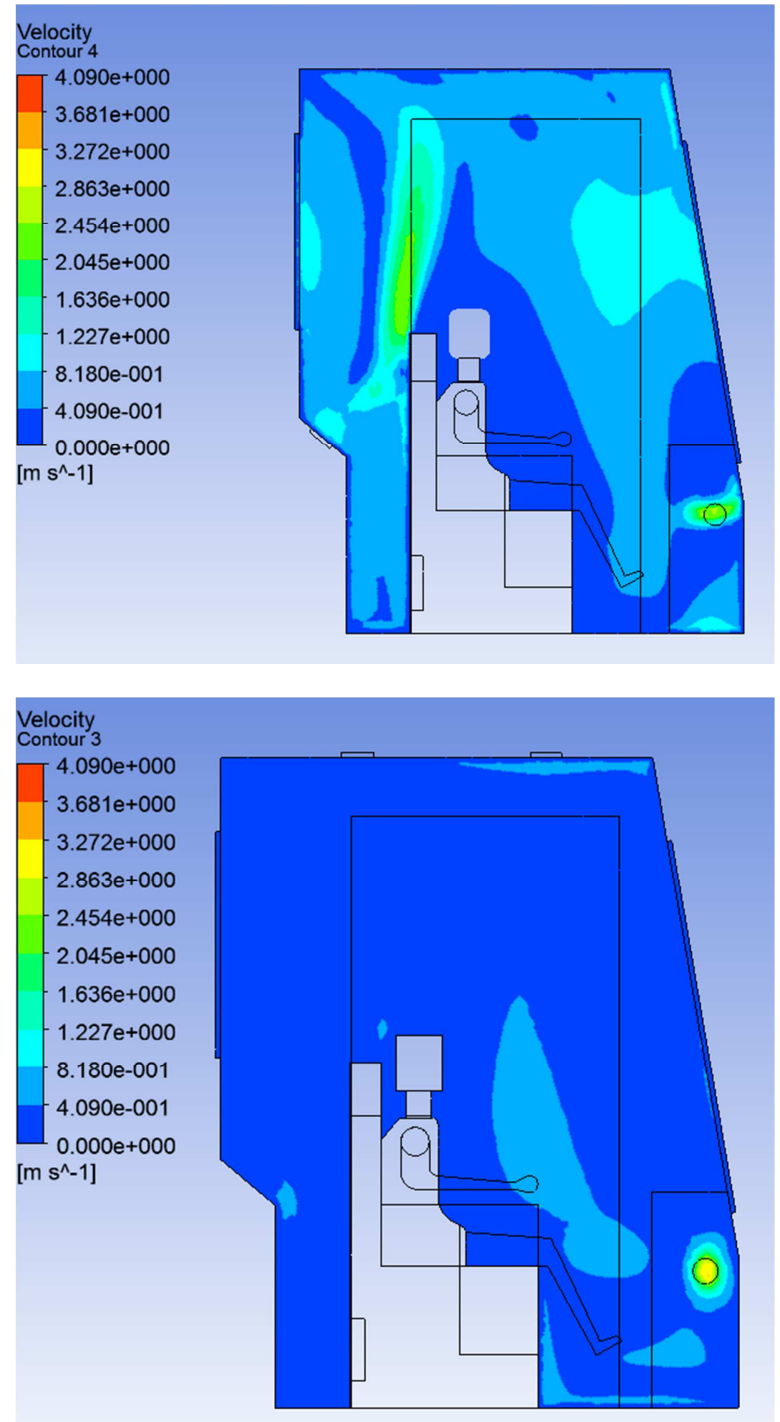

Fig. 3. The cloud map of velocity field of wind-supply forms of the side wall /top.

When the air inlet was arranged on the side wall, the airflow in the driving chamber was more uniform, which is due to the jet of the fixed air inlet collided with the airflow of the side wall. Due to the direction of the airflow, the airflow speed on the top of the cab was larger, but the air current of the driver's seat was weak. When the inlet on the top of the cab, because of the cab structure and air jet direction, the front window and the top part of the cab appeared large area where wind speed was close to zero. Due to the air jet was not too much interference with airflow of fixed outlet, made the driver wind speed around driver larger. 

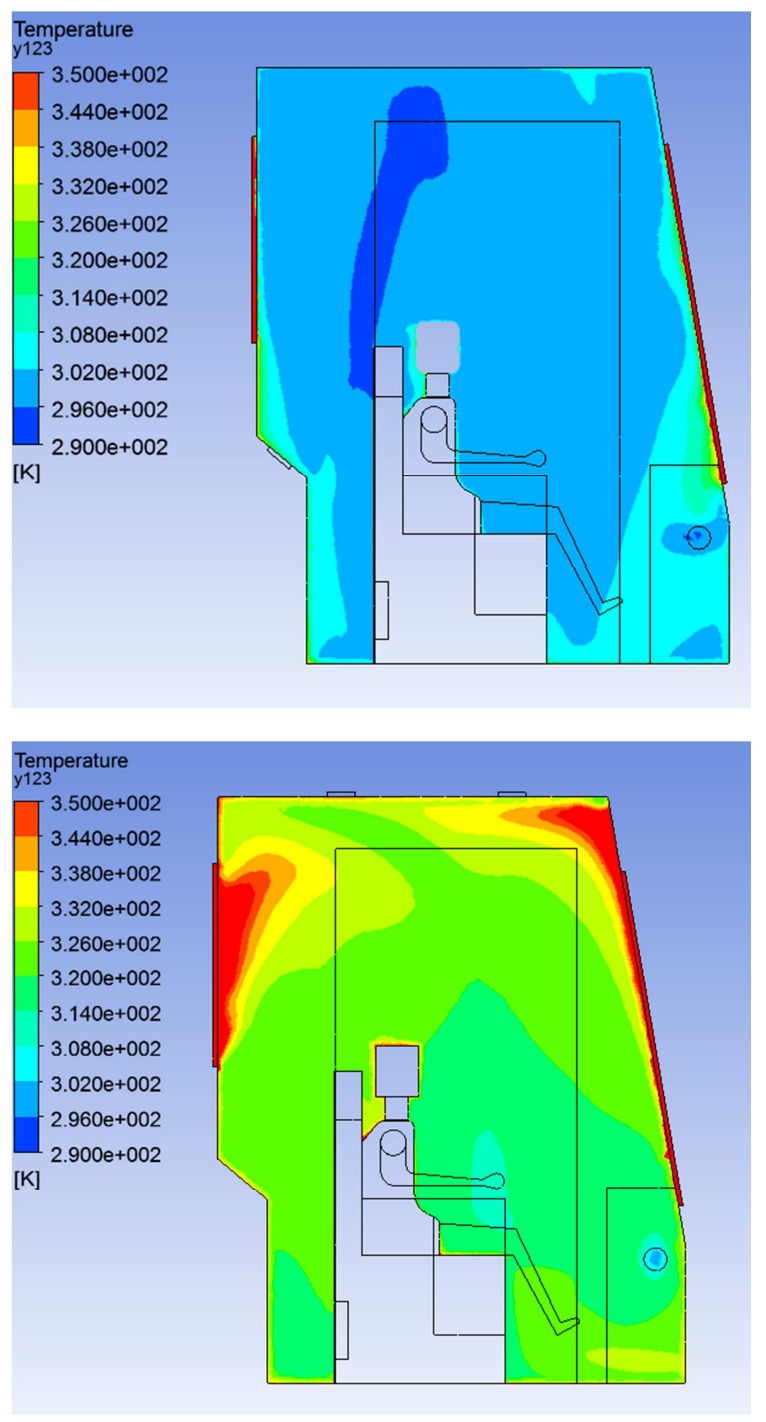

Fig. 4. The cloud map of temperature field of wind-supply forms of the side wall / top.

From Fig. 4 could be seen, the cold airflow of side wall jetted along cutting direction resulting the hot air around the top of driving room spread, produce a temperature gradient. So that, the temperature of airflow away from the side wall had corresponding high degree, and the temperature of airflow around the driver's head had suitable degree. When the inlet on the top of the cab, due to airflow jetted rapid along vertically downward, made the cold airflow rapid neutralized with hot air in driving room, which made the driving room temperature gradient was uniform, but caused the heat airflow of side wall and the front windshield squeeze, resulting in high temperature.

\section{Conclusion}

This paper used the FLUENT software to build bulldozer cab model, and we obtained the map of temperature field and velocity field of bulldozer cab under side wall and roof air-supply through $R N G k-\varepsilon$ two equation turbulence model and simple algorithm solving the governing equations.

Compared the two kinds of air-supply, we could know that driver feel better comfortable under sidewall air-supply condition, due to sidewall air-supply produce a better temperature gradient, and the roof air-supply jetted vertically downward along the driver's head caused the driver felled not suitable. Roof air-supply avoided the local airflow short, and improve the refrigeration efficiency. Specific choice which form of air supply could be based on the actual situation.

The influence of solar radiation on the flow field of the cab was very large, and the research on it will be strengthened in the future.

\section{References}

[1] Xu Li, Weng Peifen, Sun Weimin. Numerical analysis indoor air distribution and indoor air quality of three kinds of ventilation. Journal of air dynamics [J], 2003, 21 (3): 311-319

[2] CURRLE J, MAUE J, Numerical study of the influence of air vent area and air mass flux on the thermal comfort of car occupants [C]//SAE Technical Paper. 2000-01-0980

[3] Dag Aronson, Zenitha, Chtoneer, Per Elofsson. Comparison between CFD and PIV measurement in a passenger compartment. 2008

[4] Chen Jiangping, Sun Zhaopu, Que Xiongcai. Numerical simulation and experimental study on indoor ventilation of light bus, Journal of Applied Sciences [J], 2002 (02)

[5] Ye Xiaojiang. Air quality and thermal comfort of air conditioned passenger cars [J]. China Railway. 2005, (09): 26-29

[6] Lu kelong, xGU zhengqi, JIA Xinjian, et al. Analysis and improvement of the effects of air condition system on the thermal comfort of passenger compartment in a heavy truck $[\mathrm{J}]$ Automotive Engineering. 2011. 33 (2): 162-166

[7] Li C H; Han T; Koromilas C A Effects of HVAC design parameters on passenger thermal comfort 1992, 264-268

[8] Song Sihong, Yang Chen, Gou Xionglong. Numerical simulation of airflow field and temperature field of conditioned car [J]. Computer simulation. 2004 (09): 167-169

[9] Chen Zuobin. Computational fluid dynamics and applications [M]. Beijing: National Defence Industry Press, 2003

[10] Fu Dexun, Ma Yanwen. Computational fluid dynamics [M]. Beijing: Higher Education Press, 2004

[11] Xiong Kejia, Wang Wei, Zhang Wanping. Numerical calculation of flow field and temperature field in a passenger car consider solar radiation [J]. Refrigeration and air condition. 2008, 22 (6): 12-15 